\title{
Antiresonant Reflecting Optical Waveguide-Type Vertical-Cavity Surface Emitting Lasers: Comparison of Full-Vector Finite-Difference Time-Domain and 3-D Bidirectional Beam Propagation Methods
}

\author{
Nikolay N. Elkin, Anatoly P. Napartovich, Member, IEEE, Vera N. Troshchieva, Dmitry V. Vysotsky, \\ Tae-Woo Lee, Susan C. Hagness, Senior Member, IEEE, Nam-Heon Kim, Student Member, IEEE, \\ Ling Bao, Student Member, IEEE, and L. J. Mawst, Senior Member, IEEE
}

\begin{abstract}
The cold-cavity modal characteristics of an antiresonant optical waveguide-type cylindrical vertical-cavity surface-emitting laser (VCSEL) are investigated through numerical simulations using a three-dimensional (3-D) bidirectional beam propagation method (BD-BPM) and a full-vector axisymmetric finite-difference time-domain (FDTD) method. Good agreement between the BPM- and FDTD-computed radial mode profiles as well as the mode-dependent radiation losses is obtained. The results of this paper establish the accuracy of the BD-BPM technique for simulating this class of devices and confirm effective-index method predictions that antiresonance conditions for cylindrical geometry devices (i.e., VCSELs) differ from those of planar geometry devices (i.e., edge emitters).
\end{abstract}

Index Terms-Beam propagation method, finite-difference time-domain (FDTD) method, vertical-cavity surface-emitting laser (VCSEL).

\section{INTRODUCTION}

$\mathbf{V}$ ERTICAL-CAVITY surface-emitting lasers (VCSELs) possess a low-gain (the active layer thickness is tens of nanometers) and highly reflective Bragg mirrors consisting of alternating layers of quarter-wave-thick high- and lowrefractive index epitaxial materials. These lasers draw much attention due to ease of manufacturing, rather low-lasing threshold, and comparatively low-beam divergence. The ability to achieve high-power single-mode VCSELs is of great interest for many applications such as telecommunications, optical

Manuscript received August 13, 2005; revised January 4, 2006. This work was supported by the National Science Foundation (NSF) under Grant 0139823 and NSF Presidential Early Career Award for Scientists and Engineers ECS-9985004. The work of N. N. Elkin, A. P. Napartovich, V. N. Troshchieva, and D. V. Vysotsky was supported in part by the Russian Foundation for Basic Research under Project 02-02-17101.

N. N. Elkin, A. P. Napartovich, V. N. Troshchieva, and D. V. Vysotsky are with the State Research Center Troitsk Institute for Innovation and Fusion Research (TRINITI), Troitsk Moscow Region 142190, Russia (e-mail: apn@ triniti.ru).

T.-W. Lee, S. C. Hagness, N.-H. Kim, L. Bao, and L. J. Mawst are with the Reed Center for Photonics, Department of Electrical and Computer Engineering, University of Wisconsin, Madison, WI 53706-1691 USA.

Digital Object Identifier 10.1109/JLT.2006.871122 storage, and laser printing, etc. The conventional small-aperture (diameter $<4 \mu \mathrm{m})$ VCSELs are limited in a single-mode power to about $5 \mathrm{~mW}$ [1]. Attempts to increase continuous wave $(\mathrm{CW})$ output powers met the problem that a larger waveguide generally supports multiple modes. Recently, several approaches [2]-[5] exhibited about 6-mW single-mode output from large-aperture $(>8 \mu \mathrm{m})$ positive-index guided (oxide-confined) VCSELs. Another option to achieve a large spot-size single-mode operation is the usage of negative-index waveguides (antiguides) with a large built-in modal discrimination (higher order modes have significantly higher edge radiation losses). Although antiguided VCSELs have been demonstrated to be capable of operating single mode up to very high-driving currents [6]-[9], output powers were low, due to the high-edge radiation losses for the fundamental mode.

In order to reduce the edge radiation losses the reflectors can be introduced, which at the antiresonant conditions, similar to the VCSEL's longitudinal distributed Bragg reflectors (DBRs) case, minimize lateral radiation. These types of structures are generally called antiresonant reflecting optical waveguide (ARROW) [10] and have been recently proposed and demonstrated [11]-[13]. To optimize the design of this promising device, numerical modeling is of great value.

An effective index model [14] and fiber mode approximation (EI-FMA) were implemented to design a simplified ARROW (S-ARROW) [11] and an ARROW-type VCSEL [13]. In these models, the cylinder configuration was approximated by a planar geometry. Tee et al. [15] improved upon this approach by introducing the cylindrical geometry into the EI-FMA framework. They solved a two-dimensional (2-D) wave equation with the radial EI profile found by the approximate separation of vertical and in-plane variables [14]. It was shown in [15] that accounting for the cylindrical geometry leads to a down shift of the antiresonant width of the antiguided ring. An ARROW VCSEL with two cylindrical Bragg-like pairs (double ARROW) was also analyzed using the EI-FMA technique [16]. It was shown that for the double-ARROW device, the best discrimination of the fundamental mode against higher order modes is achieved when widths of low-index layers are chosen 


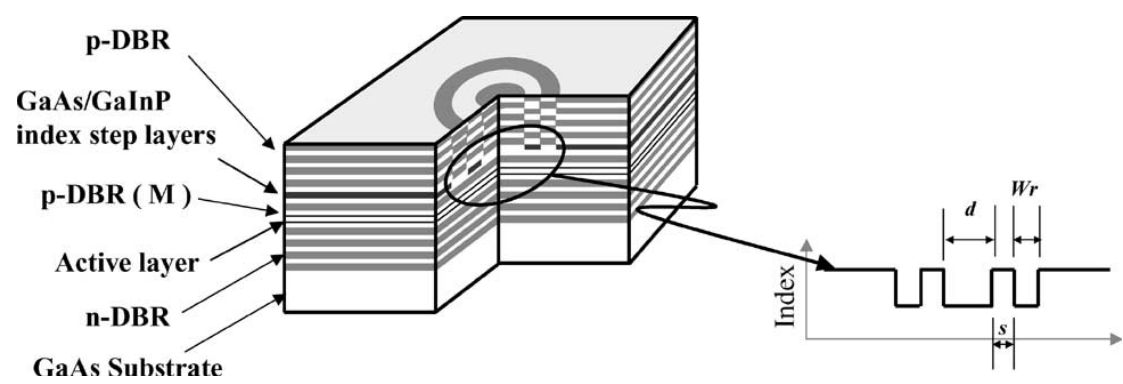

Fig. 1. Schematic diagram showing the interior cross section of an ARROW VCSEL structure.

properly. All these studies were restricted to considerations of the cold-cavity optical modes.

A recent EI-FMA analysis of an active cylindrical ARROW VCSEL [17] showed the importance of spatial hole burning (SHB) and thermal lens effects in the suppression of higher order modes. However, it is not evident that the EI-FMA is sufficiently rigorous for analyzing such effects. An alternative and more accurate approach based on the three-dimensional (3-D) bidirectional beam propagation method (BD-BPM) [18], [19] was applied to above-threshold modeling of large aperture 2-D antiguided VCSEL arrays [20]. While the BD-BPM is 10-100 times slower than the EI-FMA, it offers the potential for a greater accuracy since it is free of additional approximations required to apply the EI-FMA for above-threshold simulations. It is of interest to establish the accuracy of 3-D BD-BPM relative to more rigorous but also more computationally costly full-wave computational electromagnetics techniques.

In this paper, we investigate the accuracy of the 3-D BD-BPM in computing the cold-cavity modal characteristics of an ARROW-type cylindrical VCSEL. A full-vector axisymmetric finite-difference time-domain (FDTD) technique [12], [21] is used as the benchmark in this paper. From this paper, we can draw conclusions about the accuracy of the BD-BPM and gain confidence in the results generated from BD-BPM numerical simulations of structurally complex passive and active VCSEL devices.

\section{ARrow VCSEL DESIGN}

As shown in Fig. 1, the ARROW VCSEL is composed of transversely uniform layers forming $p$ - and $n$-DBRs and thin ring-shaped spacer layers located at some distance from the cavity, which includes three quantum wells (QWs) surrounded by spacers and cladding layers. Parameters of the simulated structure are listed in Table I. The important structural design parameters studied in the paper are 1) the number of DBR pairs $M$ separating the $\lambda$-cavity and the spacer layers that form the built-in index step; 2) the low-index core diameter $d$; and 3) the high-index reflector width $s$.

\section{FDTD METHOD}

Full-vector FDTD solutions of Maxwell's equations in cylindrical coordinates [21] are useful for investigating a transverse mode selectivity in complex VCSEL structures, as demonstrated previously for air-post index-guided and S-ARROW
VCSELs [12], [22]. The FDTD method permits a highly accurate analysis of the resonant frequencies, quality factors $(Q)$, which are inversely proportional to the mode-dependent lateral-radiation losses, and the transverse modal profile at any resonant frequency. Since the ARROW VCSEL exhibits rotational symmetry, the azimuthal $(\phi)$ dependence of the vector field components can be expressed as a Fourier series and accounted for analytically, eliminating the need for gridding in the $\phi$-direction. The FDTD simulation is simply rerun for each azimuthal mode of interest. In this paper, we focus on modes with an azimuthal mode number of $m=1$, since those modes are expected to exhibit the smallest radiation losses. In this case, the field variation along the azimuthal direction can be described simply by $\left\{E_{\rho}(\rho, z), E_{z}(\rho, z), H_{\phi}(\rho, z)\right\} \cos (\phi)$ and $\left\{H_{\rho}(\rho, z), H_{z}(\rho, z), E_{\phi}(\rho, z)\right\} \sin (\phi)$. The intensity pattern for this mode is independent of $\phi$. The resonant frequency and $Q$-factor of each transverse mode were obtained via spectral analysis of the time-domain impulse response of the cold-cavity VCSEL structure. To significantly reduce the simulation time required to accurately extract resonant frequencies and $s$, we processed the early-time response using a Padé/fast Fourier transform (FFT) linear extrapolation scheme [21]. The modal identity of the cavity resonance was obtained from a knowledge of the azimuthal mode number assumed in the simulation and from observations of the transverse modal profile for a single-frequency source excitation at the resonant frequency.

\section{Three-Dimensional BD-BPM}

The 3-D BD-BPM developed in [18] and [19] was applied to model the ARROW-type VCSEL structure. The 3-D BDBPM calculations provide estimates of the modal frequencies and losses, as well as modal patterns in the near- and far-fields. The scalar wave equation for optical field amplitude $U$

$$
\Delta U+k_{0}^{2}(n(x, y, z))^{2} U=0
$$

was solved numerically. Here, $k_{0}$ is the reference wavenumber; $n(x, y, z)$ is the refraction index. A short description of the method is as follows. The wave field within blocks of the uniform layers was decomposed into an angular spectrum of plane counter-propagating waves with help of the FFT technique. Then, a well-known transfer matrix method for each Fourier component of the wave field within the plane layered structure 
TABLE I

STRUCTURAL SPECIFICATIONS FOR SiMULATED VCSEL

\begin{tabular}{|c|c|c|c|c|}
\hline \multicolumn{2}{|c|}{ part of the construction } & material & $n$ & thickness, $\mu \mathrm{m}$ \\
\hline Cap layer & & GaAs & 3,512 & 0,07 \\
\hline P-DBR & 20.5pairs & $\begin{array}{c}\mathrm{Al}_{0.15} \mathrm{GaAs} \\
\text { AlAs }\end{array}$ & $\begin{array}{l}3,442 \\
3,007\end{array}$ & $\begin{array}{l}0,072 \\
0,081\end{array}$ \\
\hline Regrowt $h(\lambda / 2)$ & & GaAs & 3,512 & 0,14 \\
\hline Index step & & $\begin{array}{l}\text { GaAs } \\
\text { InGaP }\end{array}$ & $\begin{array}{c}3,512 \\
3,17\end{array}$ & $\begin{array}{l}0,006 \\
0,008\end{array}$ \\
\hline p-DBR & $2 / 3$ pairs & $\begin{array}{l}\text { GaAs } \\
\text { AlAs }\end{array}$ & $\begin{array}{l}3,512 \\
3,007\end{array}$ & $\begin{array}{c}0,07 \\
0,081\end{array}$ \\
\hline Cavity & & $\begin{array}{c}\mathrm{Al}_{0.25} \mathrm{GaAs} \\
\mathrm{GaAs} \\
\mathrm{In}_{0.18} \mathrm{GaAs} \\
\mathrm{GaAs} \\
\mathrm{In}_{0.18} \mathrm{GaAs} \\
\mathrm{GaAs} \\
\mathrm{In}_{0.18} \mathrm{GaAs} \\
\mathrm{GaAs} \\
\mathrm{Al}_{0.25} \mathrm{GaAs}\end{array}$ & $\begin{array}{l}3,366 \\
3,512 \\
3,6 \\
3,512 \\
3,6 \\
3,512 \\
3,6 \\
3,512 \\
3,366\end{array}$ & $\begin{array}{l}0,113 \\
0,01 \\
0,007 \\
0,01 \\
0,007 \\
0,01 \\
0,007 \\
0,01 \\
0,113\end{array}$ \\
\hline n-DBR & 30 pairs & $\begin{array}{l}\text { AlAs } \\
\text { GaAs }\end{array}$ & $\begin{array}{l}3,007 \\
3,512\end{array}$ & $\begin{array}{c}0,081 \\
0,07\end{array}$ \\
\hline Substrate & & $\mathrm{GaAs}$ & 3,512 & \\
\hline
\end{tabular}

[18] was applied to calculate the wave field after upward and downward round-trips starting from the spacer layer. In this way, the distributed reflection was described adequately. The thin spacer layer was treated as a screen with a transversely modulated phase advance $\varphi(x, y)$. We described transmission of the wave field through this screen by performing the inverse Fourier transform and multiplying the amplitude by an exponential factor dependent on the designed phase advance: $\exp [i \varphi(x, y)]$. Such a methodology allows us to calculate optical modes with a high rate not unattainable in other methods.

The FFT technique was used for the evaluation the 2-D Fourier transform and its inversion $\mathbf{F}^{-1}$. The spectrum of spatial frequencies of the plane waves incorporated into calculations was cut by some maximum value, which was evaluated from a compromise between the convergence problem and the accuracy of simulations.

To compute optical modes and their losses, a round-trip operator $\mathbf{P}$ was built up, which includes propagation of the wave field over the cavity, $p$ - and $n$-Bragg mirrors, and transmission through the spacer layer with a variable thickness. The procedure of constructing the round-trip operator is described in detail in [18], [19]. Thus, we have to solve the eigenproblem for the $\mathbf{P}$ operator

$$
\mathbf{P} u=\gamma u .
$$

The eigenfunctions of the operator $\mathbf{P}$ for a cold cavity device are the optical modes, and the corresponding eigenvalues $\gamma$ determine the losses: $\delta=1-|\gamma|^{2}$.

An important issue in completing the definition of the eigenproblem is the necessity to impose the correct boundary conditions. While boundary conditions at the top and bottom of the construction are evident, this is not the case for lateral boundaries. Our particular interest is to explore the so-called leaky-wave modes. This requires that the radiation experience no reflection at the lateral boundaries. The only layer, which 
has in-plane variable properties, is the spacer layer. The resonance wavelength shift in this layer according to [14] produces the required transverse index profile. Therefore, the absorbing boundary regions were added in this layer with proper extension of its sizes. The artificial absorption was introduced outside of each of the four lateral sides of the construction. The requirement that the artificial absorption has a negligible value in the region of the VCSEL design should be fulfilled. A particular care should be taken to avoid an influence of the absorption region on the wave-field reflection from the boundary. In selecting the amplitude and profile of the absorption function, we followed the authors [23].

The eigenproblem (2) can be solved in different ways. The first one is an analog of the Fox-Li iteration method by performing round-trips over the VCSEL. This approach allows us to find only one mode with the lowest losses. In the case for which the discrimination of modes is low, many iterations are required to converge on the fundamental mode. For the ARROW construction under consideration guided modes possess the lowest losses. Therefore, it is necessary to introduce an additional absorption into the ring-shaped waveguide to suppress the undesirable guided modes. In the actual VCSEL structure, absorbing QWs may be added to the spacer layers to accomplish this.

Another approach is to employ a method allowing for simultaneously computing an arbitrary number of modes. A sketch of the respective mathematical procedure is given below.

The operator $\mathbf{P}$ acts in the complex space with a high dimension $\mathcal{N}=N^{2}$ ( $N$ is the number of Fourier harmonics in wave-field decomposition for one Cartesian variable). In such a situation, a most appropriate way is to use Krylov's subspace methods for solving the eigenproblem. From practical considerations, only the few modes having the highest possible values of $|\gamma|$ are of interest. Among known methods for treatment of the eigenproblem in the Krylov subspace, we utilize an Arnoldi algorithm [24] because of its simplicity and robustness properties.

In order to start the Arnoldi iterations, we have to determine an initial function $\nu(x, y)$. The appropriate way is to specify $\nu=\mathbf{P}^{K} \psi$, where $\psi=\psi(x, y)$ is an arbitrary function, which can be considered as a seeded wave field, and $K$ is the number of round trips, which this field made. Solutions to the eigenproblem (2) of interest to us are sought within the Krylov subspace

$$
\mathrm{K}_{\Omega}(\mathbf{P}, \nu)=\operatorname{span}\left\{\nu, \mathbf{P} \nu, \mathbf{P}^{2} \nu, \ldots, \mathbf{P}^{\Omega-1} \nu\right\}
$$

Physically, this means that the preliminary $K$ iterations select such a wave-field distribution, which is composed of only modes with low losses. The number $K$ of preliminary iterations and the Krylov subspace dimension $\Omega$ are chosen empirically. Furthermore, the Gramm-Schmidt orthogonalization technique is applied to create a basis in the Krylov subspace. Projection of the operator $\mathbf{P}$ onto the Krylov subspace is realized by a Hessenberg matrix $\mathbf{H}_{\Omega}$ of $\Omega \times \Omega$ dimension. The eigenvalues $\gamma_{1}, \gamma_{2}, \ldots, \gamma_{\Omega}$ and eigenvectors $h_{1}, h_{2}, \ldots, h_{\Omega}$ of the matrix $\mathbf{H}_{\Omega}$ can be calculated using any relevant standard program. The

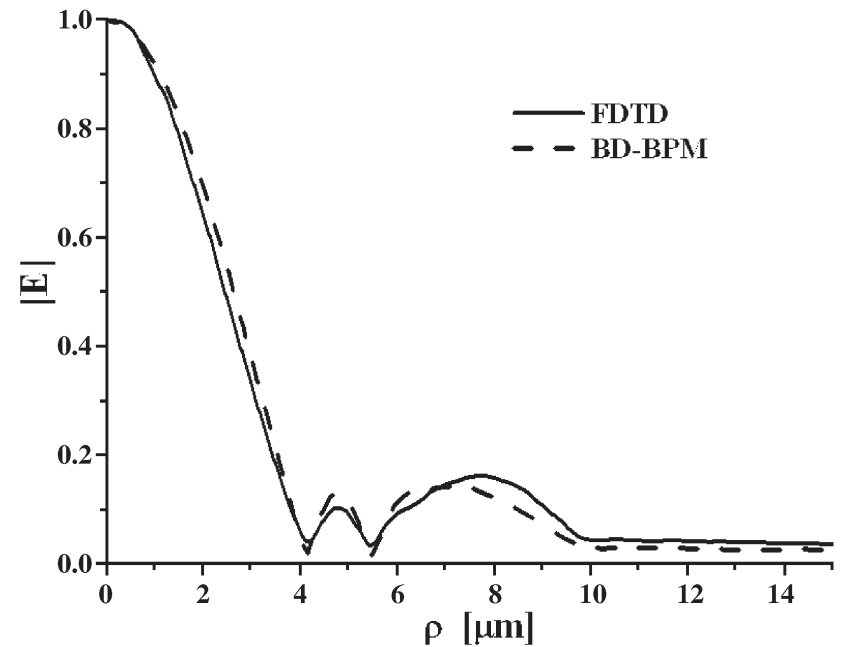

Fig. 2. Normalized amplitude of the electric field vector as a function of radial distance in the QW of an ARROW VCSEL with $M=2$ and $s=2 \mu \mathrm{m}$.

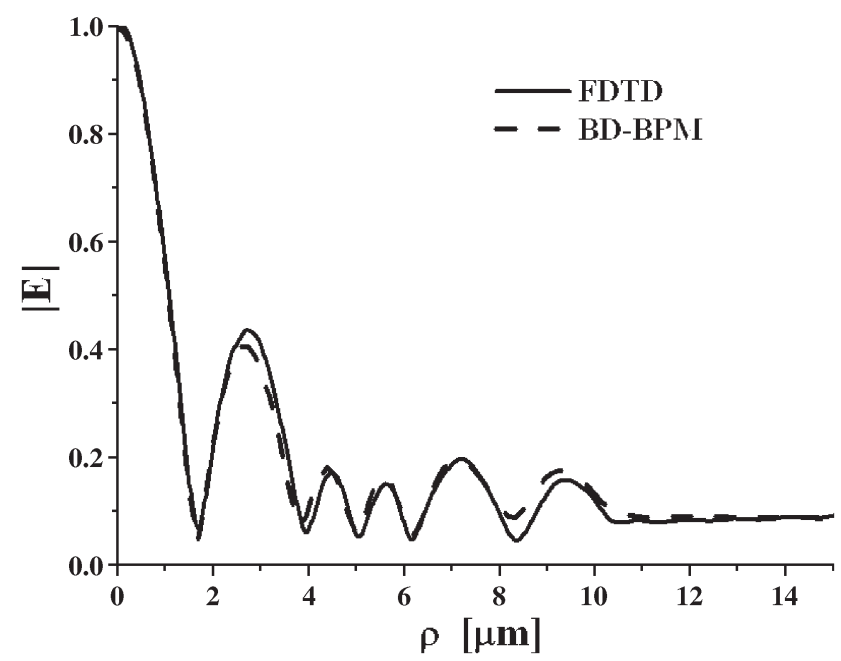

Fig. 3. Normalized amplitude of the electric field vector as a function of radial distance in the QW of an ARROW VCSEL with $M=2$ and $s=2 \mu \mathrm{m}$.

numbers $\gamma_{1}, \gamma_{2}, \ldots, \gamma_{\Omega}$ approximate the first $\Omega$ eigenvalues of the round-trip operator $\mathbf{P}$. Approximate optical modes can then be expressed as linear combinations of vectors from the orthogonalized basis with eigenvectors $\mathbf{H}_{\Omega}$ matrix weighting coefficients.

The small value of the optical mode loss sets a strict limit on the tolerances for an accuracy of a discrete approximation. The numerical scheme parameters were found from a condition of a sufficiently small loss change produced by increasing the accuracy of the calculations.

\section{COMPARISON OF RESUlts AND DisCUSSIONS}

First, we consider the radial electric-field profiles of the cavity modes. The 3-D BD-BPM simulations of a specific VCSEL structure provide a set of modes ordered in terms of increasing loss. Each BPM-computed mode was identified after pairing it with the most similar FDTD-computed mode profile. Figs. 2-5 show comparisons of the normalized 


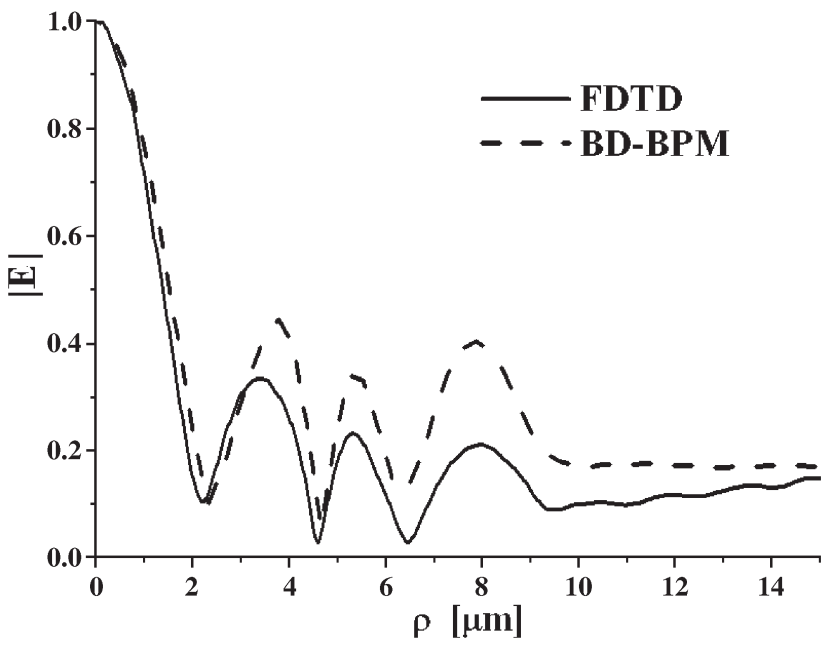

Fig. 4. Normalized amplitude of the electric field vector as a function of radial distance in the QW of an ARROW VCSEL with $M=3$ and $s=2 \mu \mathrm{m}$.

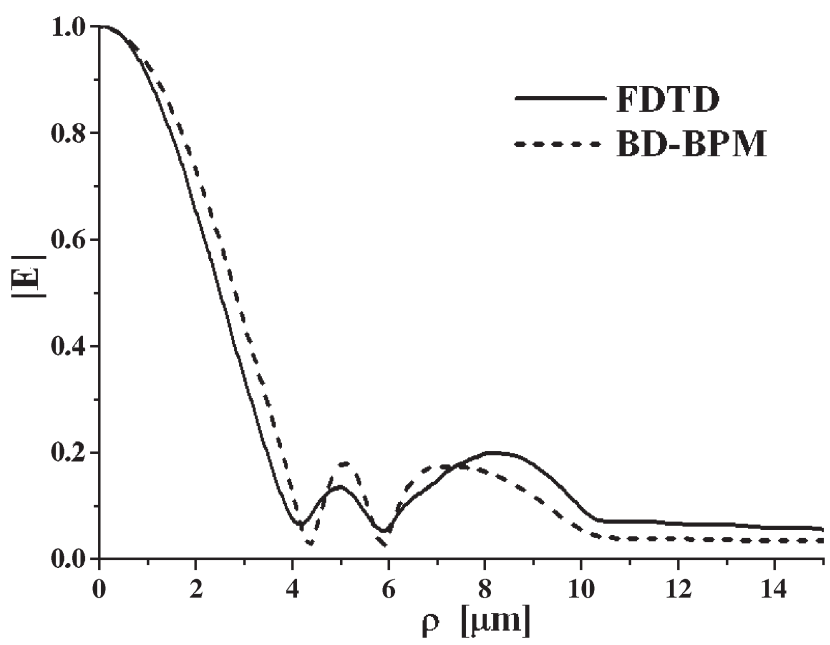

Fig. 5. Normalized amplitude of the electric field vector as a function of radial distance in the QW of an ARROW VCSEL with $M=3$ and $s=2 \mu \mathrm{m}$.

electric-field vector amplitudes of various modes as a function of radial distance in the active layer. In Figs. 2 and 3, the spacer layers, which generate the lateral index step, are located two $p$-DBR pairs away of the one-wavelength cavity (i.e., $M=2$ ), the low-index core diameter is $d=8 \mu \mathrm{m}$, and the high-index reflector widths are $s=2$ and $2.5 \mu \mathrm{m}$, respectively. In Figs. 4 and 5, the spacers are placed behind $M=3 p$-DBR pairs (i.e., the lateral index step is lower). Since the index step is lower compared with the $M=2$ design, the width of the reflector at antiresonance (i.e., lowest loss) occurs at a larger value $s=2.5$, compared with $s=2.0$ for the $M=2$ case. Figs. 2 and 5 illustrate the modal characteristics of the desired fundamental leaky mode. Figs. 3 and 4 show higher order leaky modes. As expected, these modes have much higher lateralradiation losses; hence, their amplitudes in the outer part of the construction are remarkably greater. It is seen that the BD-BPM-computed mode profiles in Figs. 2, 3, and 5 agree quite closely with the FDTD-computed distributions. For smaller $s$ values, as shown in Fig. 4, the agreement between the two calculation methods is less quantitative.

Second, we consider the radiation losses of the fundamental mode. To compare results of the simulations by the two methods, we need to find a relation between $Q$-factors given by the FDTD method and modal losses calculated by the BD-BPM. The 3-D BD-BPM simulations demonstrated that in the absence of absorption in the spacer layers, leaky modes experience remarkably greater losses than the guided modes. Therefore, a special attention was paid to identify optical modes calculated in both methods.

The $Q$-factor is inversely proportional to the decay rate of a modal wave field in the cavity. This decay rate is determined by the round-trip duration and the fraction of the radiation lost in the course of this round-trip propagation. The duration of the round trip, in turn, is proportional to an effective cavity length. The authors of [25] suggested replacing a Bragg mirror in the paraxial approximation by a plane mirror placed at a certain distance behind the front of the Bragg mirror. Generally, reflection from the Bragg mirror results in the appearance of a phase advance proportional to the deviation from the exact resonance and leads to a modification to a spherical wavefront. As a whole, the VCSEL cavity can be modeled by the equivalent Fabry-Pérot cavity [26]. For our purposes, we need an equivalent length of the cavity $L_{\mathrm{C}}^{\delta k}$ arising from the dependence of the reflection coefficient of the Bragg mirror on frequency detuning from the resonance. For the particular axial configuration with a relatively low-index contrast in $p$ - and $n$-Bragg gratings, a simplified expression for this length was derived in [19]

$$
\begin{aligned}
L_{\mathrm{C}}^{\delta k}=\left[\left(1-t_{M}\right) n_{2 M} f_{M}+t_{M}(\right. & \left.n_{2 U} f_{U}+2 n_{\mathrm{b}}\right) \\
& \left.+4 n_{\mathrm{i}}+n_{2 L} f_{L}\right] \frac{\lambda}{4 n_{\mathrm{i}}^{2}} .
\end{aligned}
$$

Here, $f_{j}=\left(n_{1 j} n_{2 j}\right)^{1 / 2} /\left(n_{2 j}-n_{1 j}\right)$ is the inverse to Bragg grating contrast and $j=U$ for upper $p$-DBR, $j=L$ for bottom $n$-DBR, $j=M$ for separating pairs of layers, $t_{M}=$ $\exp \left(-2 M f_{M}\right), n_{\mathrm{i}}$ is the refraction index in the $\lambda$-cavity, and $\lambda$ is the free-space resonant wavelength. Substituting respective values of the parameters for the constructions under study, one can find $L_{\mathrm{C}}^{\delta k}(M=2)=1.38 \lambda$, and $L_{\mathrm{C}}^{\delta k}(M=3)=1.35 \lambda$. The $Q$-factor can be expressed in terms of the mode eigenvalue $\gamma$ as

$$
Q=2 k_{0} n_{\mathrm{i}} \frac{L_{\mathrm{C}}^{\delta k}}{\delta}
$$

$\delta=1-|\gamma|^{2}$. It was found that for the fundamental mode (Figs. 2 and 5), the BD-BPM predicts losses 1.2-1.6 times higher than the FDTD simulations do. Generally, the discrepancy in losses found by two methods might be attributed to several sources: 1) an error introduced by the simplified expression (5) and 2) truncation of the angular spectrum of waves in the BD-BPM. For the ARROW construction with spacers behind two $p$-DBR pairs, both methods predict similar behavior of losses with respect to the mode order. For the 


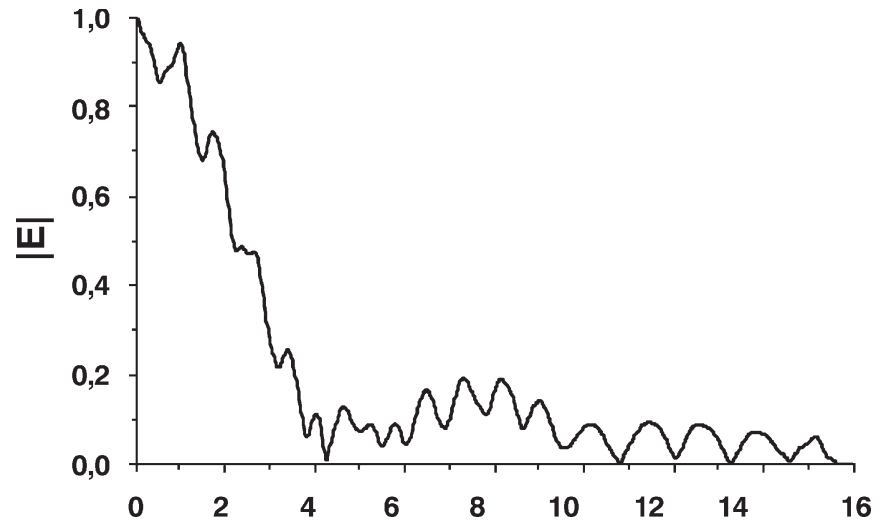

Fig. 6. FDTD-computed amplitude of the electric field vector as a function of radial distance at the top layer of an ARROW VCSEL with $s=2 \mu \mathrm{m}$ and $M=2$.

fundamental mode, the quantity $10^{4} / Q$ as predicted by the FDTD method is 0.85, while by the BD-BPM, it is 1.07. For the higher order leaky mode shown in Fig. 3, the same quantity is 7.14 (FDTD) and 6.02 (BD-BPM), respectively. Thus, we find that within the framework of the approximations for the $Q$-factor computed by the BD-BPM, only relative comparisons of the modal dependence of $Q$-factor values are possible.

It is also of interest to examine the variation of the mode radial profile along the vertical axis. Fig. 6 shows the radial mode profile at the top layer of the structure found by the FDTD method. Comparing it with Fig. 3, the mode profile at the top layer exhibits similar behavior as in the active layer but with short-period ripples indicating an interference effect. The difference in field profiles in the active layer and at the top of the device found in FDTD simulations is probably the result of scattering of the mode wave field on the annular waveguide. The scattered waves propagate at a large angle without significant interaction with the Bragg layers and experience nearly total internal reflection (TIR) from the boundary GaAs/air. The amplitude of the modal field diminishes due to reflection from the Bragg mirror at least ten times and becomes comparable with the scattered field. Taking into account that a ripple period is close to the wavelength in vacuum, this explains their formation by interference of mode wave and oblique waves incident to the surface at the angle of TIR. Oblique waves reflecting from the GaAs/air boundary escape from the device through the bottom DBR and are absorbed in the uniaxial perfect matched layer (UPML). Therefore, one may neglect the influence of these waves on optical mode characteristics.

In contrast, BD-BPM predicts nearly identical mode profiles in the active layer and at the top layer (Fig. 7). This difference between the results of the simulations by the two methods can be explained by the fact that in the BD-BPM, the spatial frequency spectrum is truncated, leading to a smoothing of the fine structure in the mode profile. Good agreement in mode profiles calculated in the active layer by both methods confirms a negligible influence of oblique waves on optical mode formation.

The consideration of the cylindrical ARROW in frames of the EI-FMA theory [15] showed that a correct account of

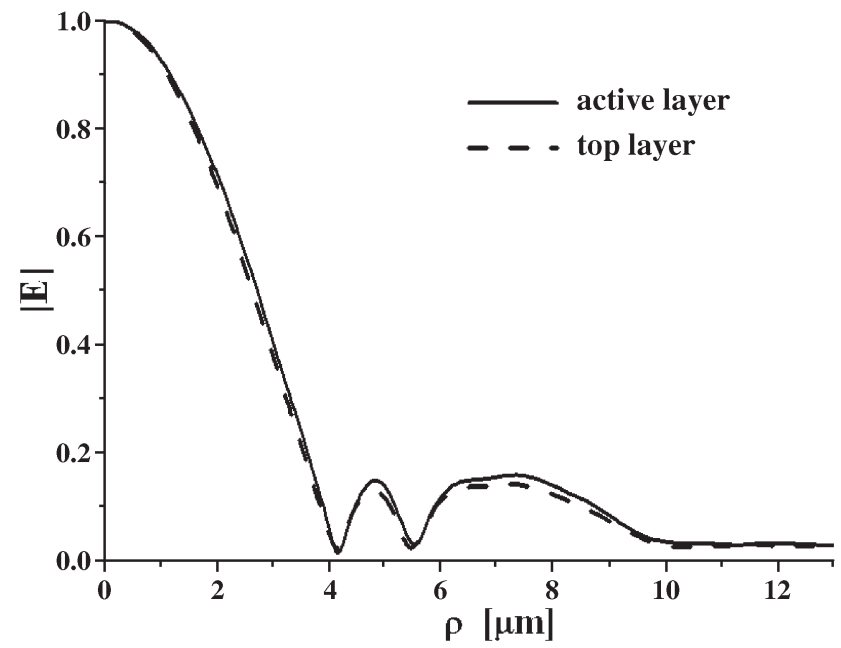

Fig. 7. BPM-computed amplitude of the electric field vector as a function of radial distance in an ARROW VCSEL with $s=2 \mu \mathrm{m}$ and $M=2$.

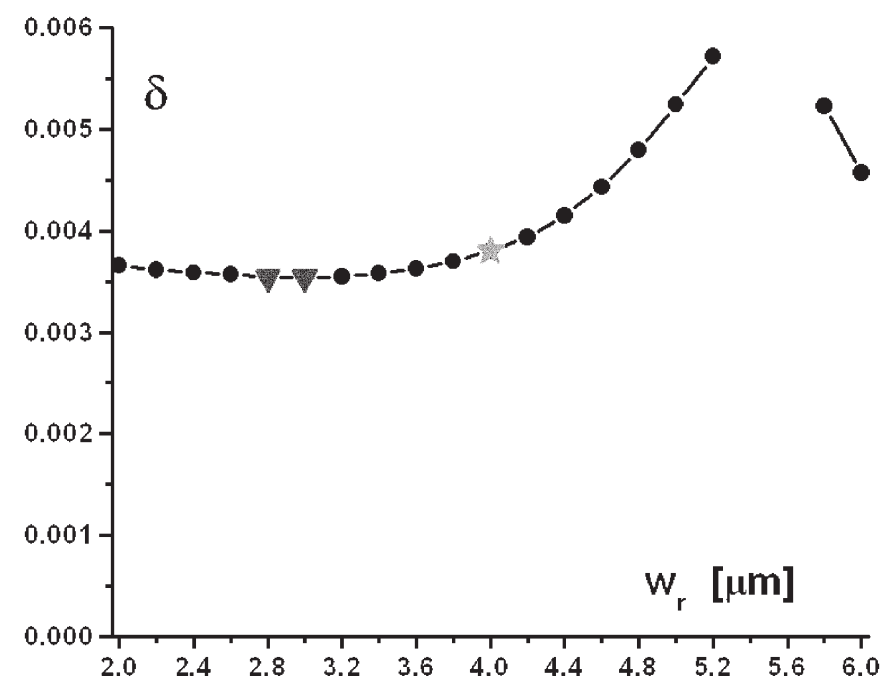

Fig. 8. Fundamental mode loss in dependence on the antiguided ring width for the construction $s=2 \mu \mathrm{m}$ and $M=2$, and $d=8 \mu \mathrm{m}$. Star designates the ring width $d / 2$, which is optimal in the planar model, and triangles indicate the ring width corresponding to the minimum losses.

cylindrical geometry leads to a shift of antiresonance condition for the width of the (low-index) antiguided ring. We applied the BD-BPM to examine this prediction. The ARROW construction with $d=8 \mu \mathrm{m}, s=2 \mu \mathrm{m}, M=2$, and variable antiguided ring width $w_{\mathrm{r}}$ was numerically studied. The calculated fundamental mode losses are displayed in Fig. 8 as a function of $w_{\mathrm{r}}$. Indeed, in agreement with the EI-FMA theory [15], Fig. 8 shows that the fundamental mode has minimum losses for the construction where the antiguided ring width is in the range between 2.8 and $3 \mu \mathrm{m}$ instead of the value $w_{\mathrm{r}}=d / 2=4 \mu \mathrm{m}$ predicted by the theory in planar geometry.

\section{CONCLUSION}

A 3-D BD-BPM was developed to calculate the optical modes of VCSELs containing antiresonant optical waveguides. 
The 3-D BD-BPM shows that the guided modes have the smallest losses in a cold cavity. A spectrum of guided and leaky modes was found for structures with a variable ring-shaped waveguide width and axial position. A number of leaky modes were calculated by the FDTD method. The radial profiles of leaky modes found by two approaches are compared, and an agreement between them is satisfactory. A tentative explanation is given of the discrepancies between modal losses calculated by two methods. The FDTD method predicts appearance of interference fringes in a mode profile at the last top layer of the device. This can be explained by the existence of waves totally reflected on the boundary top DBR air. The ARROW construction optimization was demonstrated by the 3-D BD-BPM. The external antiguided ring width giving minimum losses was found to be smaller than its value in the planar EI theory.

\section{REFERENCES}

[1] C. Jung, R. Jager, M. Grabherr, P. Schnitzer, R. Michalzik, B. Weigl, S. Muller, and K. J. Ebeling, " $4.8 \mathrm{~mW}$ single mode oxide confined top surface emitting vertical-cavity laser diodes," Electron. Lett., vol. 33, no. 21 , pp. $1790-1791$, Oct. 1997.

[2] H. J. Unold, M. Kicherer, S. W. Z. Mahmoud, R. Jager, R. Michalzik, and K. J. Ebeling, "Spatially varied anti-resonant DBR design for largearea single-mode VCSEL's," in Proc. 17th IEEE Int. Semiconductor Laser Conf., Sep. 2000, pp. 57-58.

[3] K. D. Choquette, A. J. Fischer, K. M. Geib, G. R. Hadley, A. A. Allerman, and J. J. Hindi, "High single mode operation from hybrid ion implanted/selectively oxidized VCSEL's," in Proc. 17th IEEE Int. Semiconductor Laser Conf., Sep. 2000, pp. 63-64.

[4] S. W. Z. Mahmoud, H. J. Unold, W. Schmid, R. Jager, R. Michalzik, and K. J. Ebeling, "Analysis of longitudinal mode wave guiding in vertical cavity surface-emitting lasers with long monolithic cavity," Appl. Phys. Lett., vol. 78, no. 5, pp. 586-588, Jan. 2001.

[5] A. J. Fischer, K. D. Choquette, W. W. Chow, A. A. Allerman, D. D. Serkland, and K. M. Geib, "High single mode power observed from a coupled-resonator vertical-cavity laser diode," Appl. Phys. Lett., vol. 79, no. 25, pp. 4079-4081, Dec. 2001.

[6] Y. A. Wu, G. S. Li, R. F. Nabiev, K. D. Choquette, C. Caneau, and C. J. Chang-Haisnain, "Single-mode, passive antiguide vertical cavity surface emitting laser," IEEE J. Sel. Topics Quantum Electron., vol. 1, no. 2, pp. 629-637, Jun. 1995.

[7] T. H. Oh, M. R. McDaniel, D. L. Huffaker, and D. G. Deppe, "Cavityinduced antiguiding in a selectively oxidized vertical-cavity surfaceemitting laser," IEEE Photon. Technol. Lett., vol. 10, no. 1, pp. 12-14, Jan. 1998.

[8] K. D. Choquette, G. R. Hadley, H. Q. Hou, K. M. Geib, and B. E. Hammons, "Leaky mode vertical cavity lasers using cavity resonance modification," Electron. Lett., vol. 34, no. 10, pp. 991-993, May 1998.

[9] B. S. Yoo, H. Y. Chu, M. S. Park, and E. H. Lee, "Stable transverse mode emission in vertical-cavity surface-emitting lasers antiguided by amorphous GaAs layer," Electron. Lett., vol. 32, no. 2, pp. 116-117, Jan. 1996.

[10] L. J. Mawst, D. Botez, C. Zmudzinski, and C. Tu, "Antiresonant reflecting optical waveguide-type, single-mode diode lasers," Appl. Phys. Lett., vol. 61, no. 5, pp. 503-505, Aug. 1992.

[11] D. Zhou and L. J. Mawst, "Simplified-antiresonant reflecting optical waveguide-type vertical-cavity surface-emitting lasers," Appl. Phys. Lett., vol. 76, no. 13, pp. 1659-1661, Mar. 2000.

[12] T. W. Lee, S. C. Hagness, D. Zhou, and L. J. Mawst, "Modal characteristics of ARROW-type vertical cavity surface emitting lasers," IEEE Photon. Technol. Lett., vol. 13, no. 8, pp. 770-772, Aug. 2001.

[13] D. Zhou and L. J. Mawst, "High-power single-mode antiresonant reflecting optical waveguide-type vertical-cavity surface-emitting lasers," IEEE J. Quantum Electron., vol. 38, no. 12, pp. 1599-1606, Dec. 2002.

[14] G. R. Hadley, "Effective index model for vertical-cavity surface-emitting lasers," Opt. Lett., vol. 20, no. 13, pp. 1483-1485, Jul. 1995.

[15] C. W. Tee, C. C. Tan, and S. F. Yu, "Design of antiresonant-reflecting optical waveguide-type vertical-cavity surface-emitting lasers using transfer matrix method," IEEE Photon. Technol. Lett., vol. 15, no. 9, pp. 1231-1233, Sep. 2003.
[16] C. W. Tee and S. F. Yu, "Design and analysis of cylindrical antiresonant reflecting optical waveguide," J. Lightw. Technol., vol. 21, no. 12, pp. 3379-3386, Dec. 2003.

[17] C. W. Tee, S. F. Yu, and N. S. Chen, "Transverse-leaky-mode characteristics of ARROW VCSELs," J. Lightw. Technol., vol. 22, no. 7, pp. 1797-1804, Jul. 2004.

[18] D. Zhou, L. Bao, N. H. Kim, L. J. Mawst, A. P. Napartovich, N. N. Elkin, and D. V. Vysotsky, "Modeling and observations of modal structure in antiguided VCSEL array," in Proc. SPIE, 2003, vol. 4994, pp. 112-122.

[19] N. N. Elkin, A. P. Napartovich, D. V. Vysotsky, V. N. Troshchieva, L. Bao, N. H. Kim, and L. J. Mawst, "Modeling of and experimentation on vertical cavity surface emitting laser arrays," Laser Phys., vol. 14, no. 3, pp. 378-389, 2004.

[20] L. Bao, N. H. Kim, L. J. Mawst, N. N. Elkin, V. N. Troshchieva, D. V. Vysotsky, and A. P. Napartovich, "Modeling, fabrication and characterization of large aperture two-dimensional antiguided vertical-cavity surface-emitting laser arrays," IEEE J. Sel. Topics Quantum Electron., vol. 11, no. 5, pp. 968-981, Sep./Oct. 2005.

[21] A. Taflove and S. Hagness, Computational Electrodynamics: The FiniteDifference Time-Domain Method, 2nd ed. Norwood, MA: Artech House, 2000.

[22] G. Liu, J. F. Seurin, S. L. Chuang, D. I. Babic, S. W. Corzine, M. Tan, D. C. Barnes, and T. N. Tiouririne, "Mode selectivity study of vertical cavity surface-emitting lasers," Appl. Phys. Lett., vol. 73, no. 6, pp. 726728, Aug. 1998.

[23] R. Kosloff and D. Kosloff, "Absorbing boundaries for wave propagation problem," J. Comput. Phys., vol. 63, no. 2, pp. 363-376, Apr. 1986.

[24] J. M. Demmel, Applied Numerical Linear Algebra. Philadelphia, PA: SIAM, 1997.

[25] D. I. Babić, Y. Chung, N. Dagli, and J. E. Bowers, "Modal reflection of quarter-wave mirrors in vertical-cavity lasers," IEEE J. Quantum Electron., vol. 29, no. 6, p. 1950, Jun. 1993.

[26] S. Riyopoulos, D. Dialetis, J. Ihnman, and A. Phillips, "Activecavity vertical-cavity surface-emitting laser eigenmodes with simple analytic representation," J. Opt. Soc. Amer. B, Opt. Phys., vol. 18, no. 9, pp. 1268-1284, Sep. 2001.

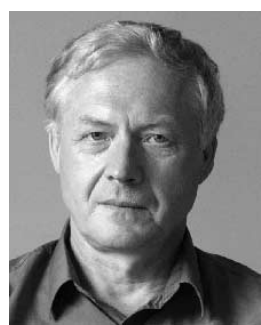

Nikolay N. Elkin was born in Kirov province, Russia, on December 10, 1951. He received the M.S. degree in physics from Moscow State University, Moscow, Russia, in 1976 and the Candidate and the Doctor of Science degrees in physics and mathematics from Moscow State University in 1980 and 1998, respectively.

His candidate dissertation was devoted to the boundary problems of the tidal waves theory. In 1979, he joined Branch of Kurchatov Atomic Energy Institute. His research concentrated on the numerical modeling of gas discharge and then on the mathematical modeling in nonlinear and resonator optics for fast-flow $\mathrm{CO}_{2}$ lasers. From 1979 to 1987, from 1987 to 1988 , and from 1988 to 1994 , he was a Junior Researcher, Researcher, and Senior Researcher, respectively, with the Branch of Kurchatov Institute. Under his active participation, the comprehensive computer models for $\mathrm{CO}_{2}$ and $\mathrm{CO}$ lasers were created and successfully used for theoretical description of these lasers. Since 1994, he is a Leading Researcher with the Troitsk Institute for Innovation and Fusion Research (TRINITI) (former Branch of Kurchatov Institute), Moscow. He has over 60 technical publications. Recently, his interests shifted to mathematical modeling in the theory of lasers with diffractive optical elements, waveguide lasers, and laser dynamics. Since 1994, he is delivering lectures in computational physics at the Moscow Institute of Physics and Technology.

Dr. Elkin received the Kurchatov Award in 1986. He is a member of the International Society for Optical Engineers (SPIE). 


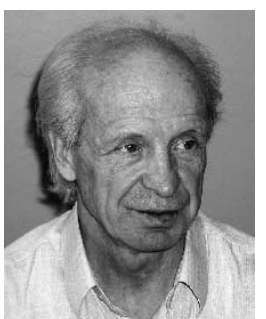

Anatoly P. Napartovich (M'03) was born in Ulan-Ude, Russia, on December 1, 1940. He received the M.S. degree in physics and engineering from the Moscow Institute of Physics and Technology, Moscow, Russia, in 1964, and the Candidate and the Dr.Sci. degrees in plasma physics from the High-Temperature Institute, Moscow, in 1969, and from Kurchatov Atomic Energy Institute, Kurchatov, Russia, in 1980, respectively.

His candidate dissertation was devoted to the resonance radiation transfer theory where for the first time in physics were introduced fractional differential equations and the concept of Levy flight. In 1967, he joined the Kurchatov Atomic Energy Institute. He predicted several kinds of plasma and optical instabilities limiting the power and optical quality of laser beams in fast-flow gas lasers. Under his guidance, the comprehensive computer models for $\mathrm{CO}_{2}, \mathrm{CO}$, and excimer lasers were formulated and successfully implemented for the theoretical description of these lasers. It was developed an analytical theory of molecular vibrations kinetics in $\mathrm{CO}$ laser. He predicted also instability of $2 \pi$-pulse propagation in two-level atomic system and initiated the work on the glow discharge in atmospheric pressure airflow. Since 1982, he is Head of the Kinetics and Optics of Low-Temperature Plasma division. Since 1986, his research concentrated on the theory of optically coupled laser arrays. He proposed a new type of coupling based on the Talbot effect, which was realized in the experiments. In 1987, he proposed optical coupling between lasers based on four-wave mixing in a nonlinear intracavity cell later realized experimentally. He also codeveloped the full analytical theory for antiguided semiconductor laser arrays in 1994. Under his guide the 3-D diffraction beam propagation code was developed for description of multicore fiber lasers. For a circular array of active cores in the fiber laser, he proposed a new construction of the spatial filter in a form of an annular waveguide embedded in a fiber with the length of a fraction of a Talbot distance. This idea was realized in the experiments performed at Max Born Institute, Berlin, Germany, in 2000. He is on the Editorial Board of Quantum Electronics. He has over 400 technical publications and holds three patents. Since 1973, he is delivering lectures on laser physics for students of Moscow Institute of Physics and Technology. Since 1984, he has held a parttime position of Professor at the Institute.

Dr. Napartovich won the State Prize in 1984 and Kurchatov Prize in 1979, 1984, and 1987. He is a member of the IEEE Lasers and Electro-Optics Society (LEOS), the Optical Society of America (OSA), SPIE, and the Laser Association.

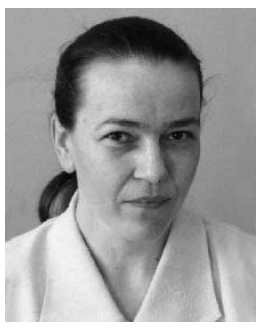

Vera N. Troshchieva was born in Grodno, Belorussia, on May 24, 1963. She received the M.S. degree in applied mathematics from the Department of Computational Mathematics and Cybernetics, Moscow State University, Moscow, Russia, in 1985.

Since then, she has been an Engineer and Researcher with TRINITI, Troitsk, Moscow region. She is engaged in numerical simulations of diffraction optics of composite laser systems (a laser controlled by injection of an external signal, a waveguide laser, multicore fiber laser, etc.). She has coauthored more than 20 technical publications.

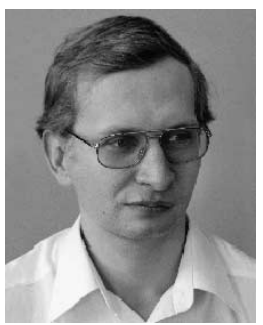

Dmitry V. Vysotsky was born in Leningrad, Russia on August 3, 1972. He received the B.S. and M.S. degrees in applied physics and the Candidate of Science degree in laser physics from the Moscow Institute of Physics and Technology, Moscow, Russia, in 1993, 1995, and 1998, respectively.

His candidate dissertation was devoted to diode laser arrays phase locking. He joined TRINITI, Troitsk, Moscow region, in 1994. Last year, his research was concentrated on phase locking of the multicore fiber laser and cavity modeling of the diode lasers: edge emitting and vertical-cavity surface emitters (VCSELs). He has over 30 technical publications.

Dr. Vysotsky has received the A. P. Alexandrov award for young researchers in 2003. He is a member of the OSA and SPIE.
Tae-Woo Lee received the B.S. and the M.S. degrees in physics from Inha University, Incheon, Korea. He received the M.S. and the Ph.D degrees in electrical and computational engineering from the University of Wisconsin, Madison, in 1999 and 2003, respectively.

In 1999, he joined the University of Wisconsin Computational Electromagnetics (UWCEM) group, where he developed and applied numerical techniques for design and optimization of optical components. Since 2003, he has been a Post Doctor in computational nanophotonics group in Argonne National Laboratory, Argonne, IL. His current research activities include theory and simulations of photon interactions in nanosystems, novel device applications of nanophotonic systems, and parallel scientific computing.

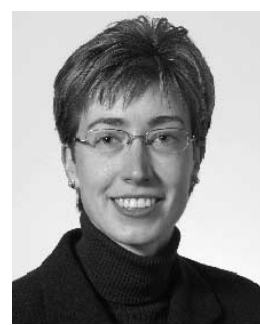

Susan C. Hagness (S'91-M'94-SM'04) received the B.S. (highest honors) and the Ph.D. degrees in electrical engineering from Northwestern University, Evanston, IL, in 1993 and 1998, respectively.

Since August 1998, she has been with the University of Wisconsin, Madison, where she is currently an Associate Professor in the Department of Electrical and Computer Engineering and a faculty affiliate of the Department of Biomedical Engineering. Her research interests include finite-difference timedomain (FDTD) theory and applications in photonics and bioelectromagnetics as well as microwave imaging, sensing, and thermal therapy techniques in biological and medical applications. She coauthored Computational Electrodynamics: The FDTD Method, 3rd. ed. (Boston, MA Artech House, 2005) with A. Taflove.

Dr. Hagness served as an elected member of the IEEE Antennas and Propagation Society (AP-S) Administrative Committee from 2003 to 2005. She is currently serving as a Member-at-Large of the United States National Committee (USNC) of the International Union of Radio Science (URSI) and as an Associate Editor for the IEEE ANTENNAS AND WIRELESS PROPAGATION LETTERS. She was the recipient of the Presidential Early Career Award for Scientists and Engineers presented by the White House in 2000. In 2002, she was named one of the 100 top young innovators in science and engineering in the world by the Massachusetts Institute of Technology (MIT) Technology Review magazine. In 2003, she received the University of Wisconsin Emil Steiger Distinguished Teaching Award. She received the IEEE Engineering in Medicine and Biology Society Early Career Achievement Award in 2004 and the URSI Isaac Koga Gold Medal in 2005. She was a National Science Foundation (NSF) Graduate Fellow and a Tau Beta Pi Spencer Fellow.

Nam-Heon Kim (S'05) was born in Seoul, Korea, in 1963. He received the B.S. and M.S. degrees in ceramic engineering from Yonsei University, Seoul, Korea, in 1986 and 1995, respectively. He is currently working toward the Ph.D. degree in electrical and computer engineering at the University of Wisconsin, Madison.

In 1989, he joined Samsung Electronics as a Researcher, where he worked on III-V lasers, including visible and electroabsorption modulator integrated distributed feedback lasers by metal-organic chemical vapor deposition (MOCVD). His current research interests include crystal growth and characterization of quantum-dot lasers and ARROW vertical-cavity surface-emitting lasers based on GaAs using MOCVD.

Ling Bao (S'05) was born in Shanxi, China, in 1975. She received the B.S. and M.S. degrees in electrical engineering from Hebei University of Technology, Tianjin, China, in 1997 and 2000, respectively. She is currently working toward the Ph.D. degree at the Department of Electrical and Computer Engineering, University of Wisconsin, Madison.

Her current research involves large-area coherent two-dimensional antiguided vertical-cavity surface-emitting laser arrays and photonic lattice structures with defect cavities. 
L. J. Mawst (M'88-SM'93) was born in Chicago, IL, in 1959. He received the B.S. degree in engineering physics and the M.S. and the Ph.D. degrees in electrical engineering from the University of Illinois at Urbana-Champaign in 1982, 1984, and 1987, respectively.

His dissertation research involved the development of index-guided semiconductor lasers and laser arrays grown by metal-organic chemical vapor deposition (MOCVD). He joined TRW Corporation, Redondo Beach, CA, in 1987, where he was a Senior Scientist in the research center, engaged in design and development of semiconductor lasers using MOCVD crystal growth. He is the coinventor of the resonant optical waveguide (ROW) antiguided array and has contributed to its development as a practical source of high-coherent power, for which he received the TRW Group Level Chairman's award. He developed a novel single-mode edge-emitting laser structure, known as the antiresonant reflecting optical waveguide (ARROW) laser, as a source for coupling high powers into fibers. He has also been involved in the development of twodimensional (2-D) coherent surface-emitting arrays, vertical-cavity surface emitters, and distributed-feedback laser structures. He is currently a Professor in the Electrical and Computer Engineering Department, University of Wisconsin, Madison, where he is involved in the development novel III/V compound semiconductor device structures, including VCSELs, active photonic lattice structures, InGaAsN lasers, and high-power Al-free diode lasers. He has authored or coauthored more than 140 technical papers and holds 18 patents. 